\title{
Are Outcomes in Congenital Cardiac Surgery Better Than Ever?
}

\author{
Ryan Holcomb ${ }^{1}$ and Akif Ündar ${ }^{1}$ \\ ${ }^{1}$ Penn State Health Department of Surgery
}

December 6, 2021

\begin{abstract}
Background and Aim of the Study: Congenital heart disease is the most common congenital defect among infants born in the United States. Within the first year of life, 1 in 4 of these infants will need surgery. Only one generation removed from an overall mortality of $14 \%$, many changes have been introduced into the field. Have these changes measurably improved outcomes? Methods: The literature search was conducted through PubMed MEDLINE and Google Scholar from inception to October 31, 2021. Ultimately, 78 publications were chosen for inclusion. Results: The outcome of overall mortality has experienced continuous improvements in the modern era of the specialty despite the performance of more technically demanding surgeries on patients with complex comorbidities. This modality does not account for case-mix, however. In turn, clinical outcomes have not been consistent from center to center. Furthermore, variation in practice between institutions has also been documented. A recurring theme in the literature is a movement towards standardization and universalization. Examples include mortality risk-stratification that has allowed direct comparison of outcomes between programs and improved definitions of morbidities which provide an enhanced framework for diagnosis and management. Conclusions: Overall mortality is now below $3 \%$, which suggests that more patients are surviving their interventions than in any previous era in congenital cardiac surgery. Focus has transitioned from survival to improving the quality of life in the survivors by decreasing the incidence of morbidity and associated long-term effects. With the transformation towards standardization and interinstitutional collaboration, future advancements are expected.
\end{abstract}

\section{Hosted file}

10-31-21 Revision of Mortality and Morbidity Review.docx available at https://authorea.com/ users/449807/articles/548279-are-outcomes-in-congenital-cardiac-surgery-better-than-ever 